\title{
Students' difficulties with integration in electricity
}

\author{
Dong-Hai Nguyen and N. Sanjay Rebello \\ Department of Physics, Kansas State University, Manhattan, Kansas 66506, USA
}

(Received 30 January 2011; published 28 June 2011)

\begin{abstract}
This study investigates the common difficulties that students in introductory physics experience when solving problems involving integration in the context of electricity. We conducted teaching-learning interviews with 15 students in a second-semester calculus-based introductory physics course on several problems involving integration. We found that although most of the students could recognize the need for an integral in solving the problem, they failed to set up the desired integral. We provide evidence that this failure can be attributed to students' inability to understand the infinitesimal term in the integral and/or failure to understand the notion of accumulation of an infinitesimal physical quantity. This work supports and extends previous research on students' difficulties with integration in physics.
\end{abstract}

DOI: 10.1103/PhysRevSTPER.7.010113

PACS numbers: $01.50 . \mathrm{Zv}$

\section{INTRODUCTION}

Students in calculus-based physics courses are often expected to have sufficient mathematical knowledge and skills to be applied to physics problems. Yet research in physics problem solving indicates that students' transfer from mathematics to physics does not happen as often and easily as we expect. This is not because students do not have the necessary mathematical resources but because they cannot appropriately activate those resources in physics contexts [1,2].

Integration is a very powerful mathematical tool widely used in physics, especially in electricity and magnetism $(\mathrm{E} \& \mathrm{M})$. Many problems in E\&M require extensive application of integration. In this study, we take a close look at students solving electricity problems involving integration to detect the difficulties students encounter when applying the integral concept in physics problem solving.

While the study focuses on students' difficulties with integration, we do not describe how consistent students were with the same incorrect reasoning. The reason we decided not to pursue this kind of longitudinal analysis was because in the teaching-learning interviews we provided students with hints to enable them to eventually solve each problem. Thus, their reasoning on the subsequent problems might have been affected. Therefore, measurements of the consistency of students' difficulties across interviews would not be valid.

In the next section, we provide an overview of the related literature on students' difficulties with integration in calculus and in E\&M. Section III describes the context and methodology of the study. The findings from the interviews are presented in Sec. IV. A discussion on how our

Published by the American Physical Society under the terms of the Creative Commons Attribution 3.0 License. Further distribution of this work must maintain attribution to the author(s) and the published article's title, journal citation, and DOI. findings support and extend other works on related topics is presented in Sec. V. The limitation of the study as well as future work will be discussed in Sec. VI.

\section{RELATED LITERATURE}

Research on students' application of calculus in physics suggested that students might not conceptually understand mathematical processes although they could easily carry out the calculations [3]. Among the earliest research on students' understanding of integration was the work of Orton [4]. In that study, 110 British students aged 16-22 were interviewed on several tasks involving the concepts of limit and integration. Orton found that students' errors with these basic concepts of calculus could be classified as structural (fundamental or conceptual), executive (operational and procedural), or arbitrary. He also found that the majority of students did not view the integral as the limit of a Riemann sum and talked about such limit as an approximation, not as an exact answer, although they had no difficulty evaluating a given Riemann sum.

Yeatts and Hundhausen [5], based on their teaching experience, described students' difficulties when transferring from calculus to physics in three categories. The first category- "notation and symbolism"-included difficulties that arose from students' rote memory of, and hence, reliance on the symbols used in each context. Mathematics and physics might use the same notation or symbol to mean different things, thus causing difficulties to students. The second category-"the distraction factor"-occurred when the surface features of the problem hindered the underlying mathematical process. The third category was "compartmentalization of knowledge," which occurred when students stored knowledge of different disciplines in different "cabinets" and activated knowledge in each "cabinet" only in the corresponding discipline.

Grundmeier et al. [6] surveyed 52 students who had completed a calculus class that covered the theory and 
techniques of integration to explore students' ability to give a definition of the definite integral in words and in symbols, to interpret and represent an integral graphically, to evaluate integrals, and to recognize the use of integrals in the real world. They found that students' knowledge of the definition of the integral did not affect their ability to perform routine calculation on the integrals. They also found that students could "perform integration as a procedure with limited understanding that they are finding the area under the curve and that this area is being found as a limit of estimation for that area."

Thompson and Silverman [7] pointed out that, for students to perceive the area under a curve as representing a quantity other than area (e.g., velocity, work), it was important that students considered the quantity being accumulated as a sum of infinitesimal bits that were formed multiplicatively. They also proposed the accumulation model which considered integration as an accumulation of the bits that were made of two multiplicative quantities. This model emphasized the two "layers" of integration: the multiplicative layer when the bits were formed and the accumulative layer when the bits were accumulated. In our study, we found evidence of students' failure in interpreting the meaning of the area under the curve when they did not understand the structure of the Riemann sum. The hints we provided to the students to help them set up the correct integrals were also built upon the structure of the Riemann sum.

Cui et al. [2] investigated students' retention and transfer from calculus to physics. They found that students had significant difficulties distinguishing variables and constants in an integral as well as determining the limits of an integral. They also found that four out of seven interviewees recognized the use of integral in a physics problem by recalling the strategy they had learned from in-class examples while the other three students had a rough idea of an integral as a sum of an infinite number of small elements.

Meredith and Marrongelle [8] investigated the resources that students used to cue integration in electrostatics problems. They used the notion of Sherin's symbolic forms [9] to describe these resources. A symbolic form is a cognitive mathematical primitive which allows students to "associate a simple conceptual schema with an arrangement of symbols in an equation" ([9], p. 482). Meredith and Marrongelle identified three symbolic forms that students used to cue integration, namely, the recall cue, the dependence cue, and the parts-of-a-whole cue. Recall is not a symbolic form because it does not have a mathematical structure, but it is commonly used in cueing integration. The recall cue is identified when students recall a previously learned strategy when solving a problem. The dependence symbolic form is described as "a whole depends on the quantity associated with an individual symbol." The dependence cue is identified when students decide to integrate because there is a quantity that depends on another quantity. The parts-of-a-whole symbolic form is described as "amounts of generic substance, associated with terms that contribute to a whole." Interpreting an integral as an accumulation of infinitesimally small elements indicates the use of parts-of-a-whole cue. Meredith and Marrongelle also found that the dependence cue was more commonly used by students than the parts-of-awhole cue, although "the use of the dependence symbolic form led to inaccuracies if the quantity being integrated was not a rate or a density" ([8], p. 577). They suggested that the parts-of-a-whole symbolic form was a more powerful and flexible resource to cue integration. They also proposed instructional strategies to promote students' use of the parts-of-a-whole recourse to cue integration in physics problems.

Most recently, Wallace and Chasteen [10] found that part of students' difficulties with Ampère's law was due to students not viewing the integral in Ampère's law as representing a sum, which aligned with the work of Manogue et al. [11] on the same topic.

In our point of view, the application of integration in a physics problem can be divided into four steps:

Step 1: recognize the need for an integral.

Step 2: set up the expression for the infinitesimal quantity.

Step 3: accumulate the infinitesimal quantities.

Step 4: compute the integral.

The work by Meredith and Marrongelle [8] investigated the first step. Although they did mention that students might misapply the symbolic forms in setting up an integral, they did not investigate this misapplication in detail. The work of Cui et al. [2] mentioned some of the difficulties students had when applying integral in physics (i.e., step 2) but did not discuss them in detail. Our current study adds the missing piece to the picture. We investigate students' difficulties in all four steps of the process, especially those in steps 2 and 3. Specifically, we examine the research question: What are the common difficulties that students encounter when solving problems in electricity involving integration?

\section{METHODOLOGY}

In the spring semester of 2009, 20 students at a large Midwestern U.S. university were randomly selected from a pool of 102 volunteers enrolled in a first-semester calculusbased physics course (which we call Engineering Physics 1 or EP1) to participate in our study on problem solving in mechanics. In that study, each of these 20 students was interviewed four times during the spring 2009 semester (interviews 1-4). In the fall semester of 2009, 15 of these 20 students, who were enrolled in the second-semester calculus-based physics course (Engineering Physics 2 or EP2) at that time, agreed to continue with our study in electricity and magnetism. Among these 15 students, there 
were 9 males and 6 females. By the time of the interviews, all of these students had completed two semesters and were taking the third semester of college calculus.

Each of these 15 students went through another sequence of four interviews (interviews 5-8) during the fall 2009 semester. Each interview occurred within two weeks after the students had completed an exam in their EP2 course. The materials covered in the interviews were the materials that had been tested in the most recent exams. All of the interviews were conducted by the first author of this paper. The interviews that we used are called teaching-learning interviews [12-14]. Unlike more commonly used clinical interviews where the goal is to probe students' reasoning, the focus of a teaching-learning interview is to find out not just how students reason, but also how students might change the ways in which they reason based on scaffolding and hints provided by the interviewer. Thus, teaching-learning interviews are used to find out how people learn and how such learning can be facilitated. In this spirit, students when presented with a problem were asked to think aloud as they attempted to solve the problem on their own. If the student was unable to proceed, or was clearly headed in a wrong direction, after a while the interviewer would interject by asking the student leading questions and providing hints to enable the student to progress toward the solution of the problem.

In each of the four one-hour interviews, students were asked to solve three to five problems on a topic in electricity and magnetism. The topics included charge distribution and electric field in interview 5, resistance and capacitance in interview 6, current density and Ampère's law in interview 7, and $R L C$ circuit in interview 8 . These problems spanned a broad range of difficulty and required several different mathematical skills. In this paper, we only discuss the problems involving integration. The problem statements will be presented in the results and discussion section when students' difficulties in solving the problems are discussed. Students' difficulties with integration in Ampère's law require in-depth studies on this topic, which have been completed by Manogue et al. [11] and Wallace and Chasteen [10], so we will not discuss students' difficulties with integration in Ampère's law in this paper.

All interviews were videotaped and audiotaped and were transcribed verbatim. Students' worksheets as well as interviewer's field notes were also collected. We first examined the field notes to identify interesting points in each interview then referred to the student's worksheet and interview transcripts for details on what students wrote and said. We focused our attention on how students recognized the need of an integral and how they set up and computed the desired integral. We listed all errors students made and the number of students making each error, and the hints provided by the interviewer, if any. Finally, we looked for the most common errors and the emergent themes.

\section{RESULTS}

Many problems in E\&M involve calculating a physical quantity from other nonconstant quantities. Unlike typical problems in calculus courses in which students are given integrals to compute, physics problems usually do not have predetermined integrals and even do not indicate that integrals are needed to solve the problems. Hence, students must be able to recognize the need for an integral and set up the desired integral from the physics scenario described in the problem statement. So the first important step in solving a problem is to recognize whether or not a problem requires integration. This step is not trivial for most students because they usually apply the formulas from the textbook without noticing the conditions under which those formulas hold. For example, the formula of resistance $R=\rho \frac{L}{A}$ only holds for a conductor with constant resistivity $\rho$ and constant cross-sectional area $A$ along its length $L$, so if $\rho$ or $A$ or both of them are not constant, then an integral must be employed to calculate resistance. Research by Meredith and Marrongelle [8], as mentioned in the literature review, reveals the resources that students invoke to cue integration.

The central idea underlying the integral is accumulation-adding up infinitesimal amounts of a physical quantity to obtain the total amount of that quantity (e.g., resistance) or adding up infinitesimal effects to obtain the total effect (e.g., electric field). So to obtain a correct integral, students must have the correct expression for the infinitesimal elements and add up those elements in an appropriate manner (e.g., vectorially, reciprocally). An integral is ready to be computed only after all these steps are done correctly. In summary, the application of integration in physics problems can be divided into four steps: (i) recognize the need for an integral, (ii) set up the expression of the infinitesimal elements, (iii) accumulate the infinitesimal elements, and (iv) compute the integral.

A common theme observed in our interviews was that all students, at some point during the interviews, expressed their understanding of an integral as an accumulation of infinitesimal elements. However, only one or two of them could carry out this strategy without assistance from the interviewer. All other students were not confident in performing the steps and needed guidance through the process.

In each of the following subsections, we discuss the difficulties students encounter at each of the steps mentioned above. At each step, we will start with a general description of the difficulties and then present examples of those difficulties in each of the problems under investigation as well as the number of students making each error. We will use pseudonyms for the students mentioned in these subsections. 


\section{A. Students' recognition of integration}

Most of the students in our interviews did not have significant difficulty recognizing the need for integration in solving the problems. We observed that the nonconstant physical quantity given in the problem statement was the major cue for integration, while recalling similar situations was the strategy used by a few students. These findings are consistent with those of Meredith and Marrongelle which state that the recall cue and the dependence cue are the most common cues used by the students to cue integration in electrostatic problems. So in this subsection, we will describe how our students recognized the use of integration in our interview problems and also relate with the findings of Meredith and Marrongelle in their study.

The charged arch problem (Fig. 1) and the charged rod problem (Fig. 2) were asked in interview 5. These problems were very similar to the homework and exam problems in the course, so all students stated that they had to set up an integral to calculate the electric field. In other words, the recall cue was used by the students to cue integration in these familiar problems.

On the cylindrical conductor problem (Fig. 3), 12 out of 15 students stated, with different levels of confidence, that an integral was needed because the resistivity was changing along the conductor. The reasoning provided by David "since $\rho$ isn't constant we're going to have to do an integral" was typical for students who were confident with their reasoning. On the other hand, the question posed by Mary, after setting up the expression $\frac{\alpha x L}{A}$, "Do I have to put an integral somewhere?" indicated her uncertainty about the use of integration in the problem. The remaining three students also arrived at the expression $\frac{\alpha x L}{A}$ but stated that was the final answer. When the interviewer hinted that the final answer should not contain $x$, these students were able to recognize that they needed an integral. The following excerpt is typical among this group of students.

You are standing at the center of a non-conducting circular arch of radius $\mathrm{R}$ in a stormy day. There are negatively charged clouds over the arch. The charge distribution $\lambda$ on the arch now depends on the angle $\theta$ as per the function:

$$
\lambda(\theta)=\lambda_{0} \cos \theta
$$

where $\lambda_{0}$ is a positive constant.

Find the magnitude and direction of the electric field at your feet (i.e. at a point $O$ on the ground directly below the top of the arch).

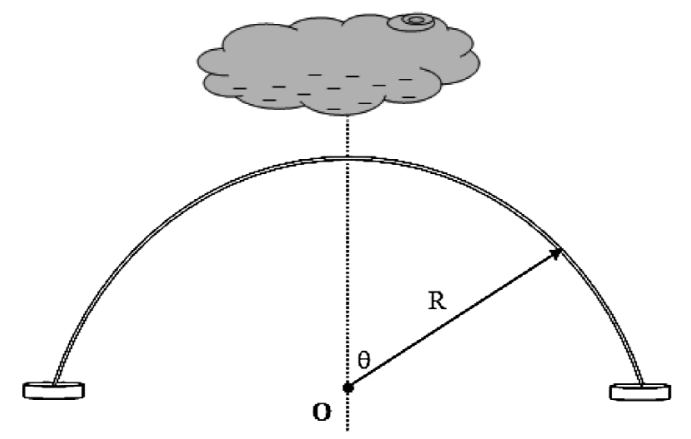

FIG. 1. The charged arch problem (interview 5).

A straight metal rod of length $L$ is lying on the ground but is insulated from the ground. The charge on the rod is distributed with charge density given as per the following function:

$$
\lambda(x)=\alpha x^{2}
$$

where $\alpha$ is a positive constant, $x$ is the position on the $\mathrm{X}$-axis relative to the origin $\mathrm{O}$ as shown in the figure below.

Find the magnitude and direction of the electric field at your feet, located at $x=0$.

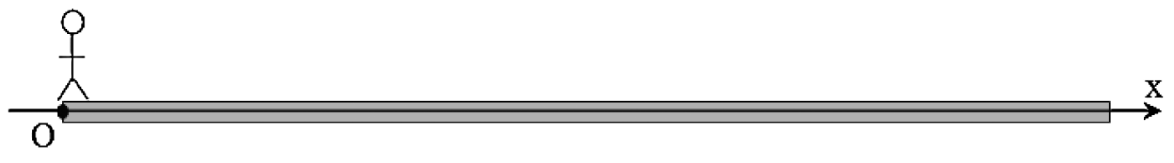

FIG. 2. The charged rod problem (interview 5). 
Find the resistance of a cylindrical conductor of length $L$, diameter $D$. The resistivity $\rho(x)$ is changing along the conductor as per the following function:

$$
\rho(x)=\alpha x
$$

where $x$ is the distance from the left end of the conductor.

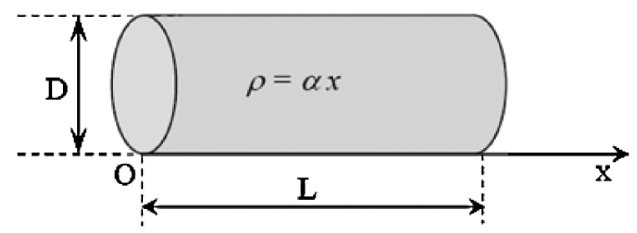

FIG. 3. The cylindrical conductor problem (interview 6).

Interviewer: Is this $\left[\frac{\alpha x L}{A}\right]$ your final answer?

Brian: Uh ... yes.

Interviewer: But that answer contains $x$ which is changing.

Brian: Okay ... so ... should I use integration?

The cylindrical problem was not asked on any homework or exam in the course, so the recall cue was not employed. Instead, the majority of students recognized the need for an integral based on the nonconstant resistivity that was given; i.e., they employed the dependence cue in this problem. Guiding the students to think of the dependence cue was the strategy employed by the interviewer to hint students who did not spontaneously recognize the use of the integral as in the excerpt above.

The truncated-cone conductor problem (Fig. 4) followed the cylindrical conductor problem in interview 6 . Thirteen out of 15 students were able to recognize comparing this problem with the cylindrical conductor problem. They stated that they could use the integral set up in the cylindrical conductor problem except that the area was then a variable. The following excerpt demonstrates this reasoning.

Interviewer: Let's move on to the next problem [the truncated-cone conductor problem]

James: Alright. The tiny bit in $R$ equals constant $\rho$ times change in $L$ over change in area [writes $d R=\frac{\rho d L}{A}$, then replaces $A$ with $\frac{1}{4} \pi D^{2}$ ] so basically this is the same integral as in the problem we've just done. This one now has two variables. We're not summing any changes in diameter, we're just summing the length pieces. Um so ... [writes $\left.R=\frac{\rho}{\frac{1}{4} \pi} \int \frac{d L}{D^{2}}\right] \ldots$ little $d$ and big $D$, so we have to incorporate that in there somehow.

The other two students wrote an integral with $d A$ - the infinitesimal cross-sectional area-as the infinitesimal term. This error will be discussed in Sec. IV B because it is related to the expression of the infinitesimal quantity.

The capacitor problem (Fig. 5) was the last problem of interview 6. Only 12 out of 15 students got to this problem within the one-hour time limit of the interview. All of them stated that they had to use integral to calculate the capacitance because the diameter was not constant. The following excerpt is typical for this reasoning.

Interviewer: Now we have the last problem. [the capacitor problem]

James: Okay ... So here we're trying to find capacitance which equals $\frac{\varepsilon_{0} A}{D}$. Diameter is not going to be constant so we are going to have $C$ equals [writes $C=\int \frac{\varepsilon_{0} A}{D}$ ] $\varepsilon_{0}$ is still constant though.

In this excerpt, the student recalled the formula for the capacitance of a parallel-plate capacitor and identified nonconstant and constant quantities. As he recognized that the diameters of the plates were not the same, he decided that the capacitance must be calculated by an integral. This is evidence of the use of the dependence cue to cue integration in this problem.

The current problem (Fig. 6) was asked in interview 7. This problem was one of the homework problems that students were asked prior to the interview. Thirteen out

A conductor has diameter decreasing from $D$ to $d$ over its length $L$. The resistivity $\rho$ is constant along the length of this conductor. Find the resistance of this conductor.

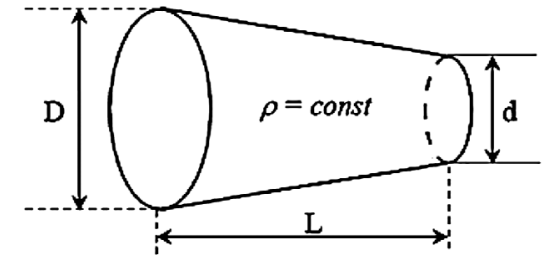

FIG. 4. The truncated-cone conductor problem (interview 6). 
A capacitor is made of two circular conducting plates of diameter $D$ and $d$. The permittivity $\varepsilon$ of the material filled between the plates is constant. Find the capacitance of this capacitor.

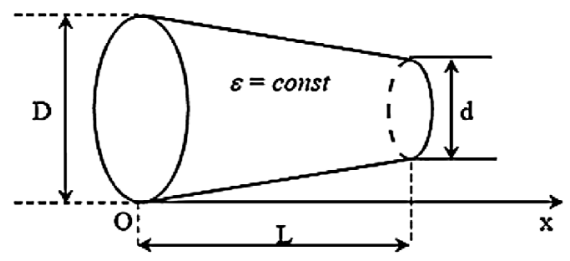

FIG. 5. The capacitor problem (interview 6).

A cylindrical wire of radius $R$ is carrying a current of density $j=\alpha \cdot r$ ( $\alpha$ is a constant, $r$ is the distance from the center of the wire). Find the total current in the wire.

\section{$j=\alpha \cdot r$}

FIG. 6. The current problem (interview 7).

of 15 students stated that they needed to have an integral to calculate the total current. These students recognized the use of integral by recalling the homework problem or reasoning on the nonconstant current density, i.e., both the recall cue and the dependence cue were used by the students in this problem. The other two students attempted to find the total current by multiplying the current density at the surface of the wire by the total cross-sectional area of the wire. Upon being hinted that the current density had different values at different distances from the center of the wire, these students stated that they had to do an integral. The following excerpt was from an interview with one of these students.

Interviewer: How do you find the total current in this problem? [the current problem]

Chelsea: Current is $j$ times $A$.

Interviewer: What value of $j$ in this problem?

Chelsea: Well $j$ is $\alpha$ times $r$, and the radius is $R$, so $j$ is $\alpha$ times $R$. [writes $I=\alpha R A$ ]

Interviewer: So what is $A$ ?

Chelsea: $A$ is ... pi $R$ squared ... [writes $I=\alpha R A=$ $\alpha R \pi R^{2}=\alpha \pi R^{3}$ ]

Interviewer: But the current density is changing as you go from the center to the edge of the wire, so it's not always $\alpha$ times $R$.

Chelsea: Oh okay ... so then I will do an integral.

Interviewer: How do you know you have to do an integral?

Chelsea: "Cause you said $j$ was changing.

In this excerpt, the student attempted to use the value of the current density at the edge of the wire to plug in the equation for current. Upon being hinted that the current density was changing, she was able to recognize the need for an integral. Her reasoning "cause you said $j$ was changing" indicates that she used the dependence cue to cue integration after the hint.

In conclusion, we found that most of the students could easily recognize the need for an integral in the problem. Students' familiarity with the problems and the presence of the nonconstant quantities were the major cues for students to think of using integration. This finding agrees with the finding of Meredith and Marrongelle that the recall cue and the dependence cue were most commonly used by students to cue integration in physics problems [8]. In our study, students usually used the recall cue in problems which were familiar to them, and used the dependence cue in unfamiliar problems. Guiding students' attention to the nonconstant quantity to trigger the dependence cue was also a productive strategy used by the interviewer to help students recognize the use of the integral when they could not invoke it by themselves.

\section{B. Set up the expression for the infinitesimal quantities}

In order to calculate an integral, one must know the variable of integration. One way to do that is to look at the infinitesimal term (e.g., $d x, d r, d \theta, \ldots$ ) in the integral. In physics problems, the infinitesimal term also carries a physical meaning that must be understood while setting up the integral. For example, if $F(x)$ is a function of force with respect to position $x$, then $\int F(x) d x$ means integrating the product of the force $F(x)$ at position $x$ and the corresponding infinitesimal distance $d x$, in the direction of the force to obtain the total work done over the whole distance. However, $\int F(t) d t$ means integrating the product of the force $F(t)$ at time $t$ and the corresponding infinitesimal time interval $d t$ to obtain the total impulse due to the force 
over the total time interval. In these examples, $d x$ and $d t$ not only indicate the variable of integration but also have their own physical meanings: infinitesimal distance and infinitesimal time interval. So it is mathematically incomplete and physically meaningless to write the integral as $\int F(x)$. However, it was observed that many students in our interviews either set up the integral without the infinitesimal term or simply appended it to the integrand or to whatever quantity was changing. These actions essentially changed the physical meaning of the integrand.

Charged arch problem (Fig. 1).--Starting with the formula for the electric field due to a point charge $E=$ $\frac{1}{4 \pi \varepsilon_{0}} \frac{q}{r^{2}}$, all students were able to write the electric field due to a charge element $d q$ as $d E=\frac{1}{4 \pi \varepsilon_{0}} \frac{d q}{r^{2}}$.

Charged rod problem (Fig. 2).-This problem followed the charged arch problem in the same interview. After doing the charged arch problem, all students knew that they had to integrate $d E=\frac{1}{4 \pi \varepsilon_{0}} \frac{d q}{r^{2}}$.

Cylindrical conductor problem (Fig. 3).-To solve this problem, one must set up the expression $d R=\rho(x) \frac{d x}{A}$ for the infinitesimal resistance of a thin slice of the conductor, then integrate it to find the total resistance $R=\int_{0}^{L} \rho(x) \frac{d x}{A}$, where $A$ is the constant cross-sectional area of the conductor. Eight out of 15 students started with the formula of resistance $R=\rho \frac{L}{A}$ and then set up the integral $R=\frac{L}{A} \times$ $\int \rho(x)$ or $R=\frac{L}{A} \int \rho(x) d x$. The first integral was mathematically incomplete and the second integral did not represent any physical quantity. By simply appending the infinitesimal term $d x$ into the integrand, students changed the physical meaning of the infinitesimal quantity. For example, the expression $\frac{\rho d x}{A}$ represents the resistance of the infinitesimally thin conductor whose length was $d x$, while the expression $\frac{\rho L}{A} d x$, obtained by appending $d x$ into the formula for resistance, did not represent any physical quantity. The following excerpt was taken from the interview with a student who set up the integral $R=\frac{L}{A} \int \rho(x)$ and then appended the term $d x$ into the integrand when being asked about the variable of integration.

Stephanie: So general equation [writes $R=\frac{\rho L}{A}=\frac{4 \rho L}{\pi D^{2}}$ ] and then this $[\rho]$ is a function of $x$ and $\ldots$ I have to do something with the integral because we have to go from the zero point to $L$.

Interviewer: Okay, so integral of what?

Stephanie: Of $\rho(x)$. [writes $\int \frac{4 L}{\pi D^{2}} \rho(x)$ ]

Interviewer: Uh huh. But what variable are you taking integral with?

Stephanie: Variable here is $x$.

Interviewer: So you should have an infinitesimal term to indicate that...

Stephanie: What do you mean by infinitesimal term?

Interviewer: I mean $d x$.

Stephanie: Okay. [writes $\int_{0}^{L} \frac{4 L}{\pi D^{2}} \rho(x) d x$ ] So four L over pi $D$ squared are all constants and you have the integral of $\rho(x)$ that's gonna go from $0 \ldots$ to $\ldots L$ [does the integral and gets $\left.\frac{2 \alpha L^{3}}{\pi D^{2}}\right]$

Interviewer: Okay ... Let's see, you appended $d x$ into the integrand before taking the integral. What is the meaning of $d x$ ?

Stephanie: It is the variable of the integral.

Interviewer: Yeah, right, but what is the physical quantity that $d x$ represents?

Stephanie: Physical quantity? Um ... I don't know ... It's just $d x$.

Interviewer: Alright, $d x$ is a small length segment along the conductor. With that, can you tell the meaning of the whole integral that you have?

Stephanie: Um ... going from there to there. [two ends of the cylinder]

Interviewer: No, I mean the process underlying integration.

Stephanie: Is it the adding small pieces thing?

Interviewer: Yeah, right. So in your integral, what are the pieces that you add?

Stephanie: The $x$ values ... or the cross-section values.

Interviewer: Okay, from your integral, the small piece is $\frac{4 L}{\pi D^{2}} \rho(x) d x$. Can you explain the physical meaning of this term?

Stephanie: I don't know.

Interviewer: Okay, in this expression for the piece, you have the total length $L$, resistivity $\rho(x)$, and infinitesimal length $d x$ on the numerator and area $\frac{\pi D^{2}}{4}$ on the denominator. But in the formula for resistance $R=\frac{\rho L}{A}$, there is only one length on the numerator. So the expression you have does not represent resistance of a piece of the conductor.

Stephanie: So I should remove this $L$ then.

Interviewer: Yes, because you already have the infinitesimal length $d x$.

Stephanie: Got ya.

Stephanie easily recognized that she needed to do an integral "to go from the zero point to $L$." However, the integral she set up after that did not have an infinitesimal term $d x$. She indicated that she did not know what the interviewer meant by "infinitesimal term" and simply appended it to her integral without any changes to the integrand. Her answer to the interviewer's question on the physical meaning of $d x$ - "I don't know ... it's just $d x$ "-indicated that she did not know what $d x$ represented. Even after being told explicitly that $d x$ represented an infinitesimal length segment, she was still unable to interpret the physical meaning of the expression she set up for the pieces, and therefore was unable to recognize that her expression for the infinitesimal piece did not represent the resistance of an infinitesimal conductor. This excerpt is an example of the instance that students' lack of understanding of the infinitesimal term led them to set up incorrect expressions for the infinitesimal quantity. In this case, Stephanie included both $L$ and $d x$ in the expression of 
infinitesimal resistance because she did not know what $d x$ meant in the problem.

Among the remaining seven students, one student recognized that she needed an infinitesimal length $d L$ in place of $L$ in the formula, which was correct. Three other students recognized this after being reminded that $L$ was the length of the whole conductor while we only considered the length $d x$ of an infinitesimal conductor at location $x$. The other three students did not know what to do in the problem and needed step by step instruction to solve the problem.

Truncated-cone conductor problem (Fig. 4).-Twelve out of 15 students stated that they could use the integral set up in the cylindrical conductor problem but with area being a variable. They could also recognize that since there were two variables in that integral, $x$ and $A$, they had to write one variable in terms of the other in order to integrate. The transcript presented when we discussed this problem in Sec. IVA is an example from this group of students. All of the students needed a lot of guidance on basic geometry to write the area $A$ in terms of $x$.

One student set up the correct integral but stated that the limits of integral were from $d$ to $D$ because the diameter was changing. Upon being hinted that $d x$ indicated integration with respect to $x$, hence the limits should be the range of $x$, this student recognized that the limits were from 0 to $L$. Therefore, we interpret this student's wrong choice of limits as evidence that she did not understand that $d x$ indicated the integration variable $x$.

Two other students set up the integral for resistance as $R=\int_{\pi(d / 2)^{2}}^{\pi(D L} \frac{\rho L}{d A}$. These students stated that because area $A$ was changing, they used the infinitesimal area $d A$. Obviously, the term $\frac{\rho L}{d A}$ did not represent the infinitesimal resistance of a thin slice of the conductor.

Capacitor problem (Fig. 5).-To solve this problem, students needed to think of a capacitor with a large separation between the plates as a series combination of several capacitors made of fictitious plates separated by an infinitesimal distance $d x$. This strategy was novel to many students, so they attempted to use the formula for capacitance of a capacitor with small separation. The students needed to be told that the formula was only applicable to the case when the separation was small compared to the diameters of the plates, and hence they had to consider the capacitor with a large separation as being made of several plates close to each other. Then, 10 out of 12 students were able to set up the correct expression for capacitance of a capacitor with infinitesimal separation between the plates $d C=\varepsilon \frac{A(x)}{d x}$. The other two students used the differential area $d A$ and got $d C=\varepsilon \frac{d A}{L}$. This error was similar to the error observed in the truncated-cone resistor problem, where students had $\frac{\rho L}{d A}$ as the infinitesimal resistance. This type of error suggested that these students seemed to simply prefix " $d$ " to whatever quantity that was chang- ing (i.e., area $A$ in these cases) without understanding the meaning of the infinitesimal term in the integral.

Current problem (Fig. 6).-The correct expression for the infinitesimal current in the wire is $j(r) d A$, where $j(r)$ is the current density at a distance $r$ from the center of the wire and $d A$ is the area of an infinitesimally thin ring on the cross section of the wire. Thirteen out of 15 students made mistakes similar to those observed in the cylindrical conductor problems: they set up $I=A \int j(r)$ or $I=$ $A \int j(r) d r$, where $A$ was the total cross-sectional area of the wire. This is further evidence that students seemed to integrate whatever was changing without understanding the physical meaning of the expression for the infinitesimal quantity, which usually led them to incorrect integrals. When the interviewer reminded students about the formula $I=\int j(r) d A$, all students agreed that they had seen it before but then failed to explain what $d A$ meant in that formula.

In conclusion, we found that students' failure in setting up the expression for the infinitesimal quantity was due to their lack of understanding of the physical meaning carried by the infinitesimal term (e.g., $d x, d r, d \theta, \ldots$ ) and the expression for the infinitesimal quantity. This lack of understanding caused students to ignore the infinitesimal term or to simply append it to the integrand, or even to prefix $d$ to whatever quantity was changing when setting up the expression for the infinitesimal quantity. All of these actions essentially changed the physical meaning of the expression being set up as discussed in the truncated-cone conductor, the capacitor, and the current problems above.

\section{Accumulating the infinitesimal quantities}

It was observed in our interviews that after having the correct expression for the infinitesimal quantity, almost all students started integrating that expression without attending to how these quantities should be added up.

Charged arch problem (Fig. 1).- Electric field is a vector quantity, so the electric fields $d E$ due to the infinitesimal elements of charge on the arch must be added vectorially. Eight out of 15 students in our interview did not notice the vector nature of $d E$ and integrated the whole $d E$, while the other seven students used symmetry to argue that only the $y$ component of the electric field due to each charge element contributed to the total field and integrated only the $y$ component of $d E$.

Charged rod problem (Fig. 2).-The electric fields $d E$ due to all infinitesimal elements of charge $d q$ on the rod were pointing in the same direction so the total field could be obtained by simply integrating $d E$. So even though all of the students could do this step, we could not conclude whether they understood that they were adding vectors having the same direction or were just adding the electric fields as if they were scalars.

Cylindrical conductor and truncated-cone conductor problems (Figs. 3 and 4).-The slices that made up the 
conductor were connected in series, so the total resistance could be obtained by adding up the resistance of these slices. When the thickness of each slice became infinitesimally small, this was done by integrating $d R$. Similarly, in the current problem (Fig. 6), because the currents in all thin rings that made up the cross section of the wire were in the same direction, the total current could be obtained by integrating the infinitesimal current $d I$ in each ring. In these three problems, the total quantities were obtained by simply integrating the infinitesimal quantities, i.e., $R=$ $\int d R$ and $I=\int d I$, so we could not conclude whether or not students understood how the infinitesimal quantities must be accumulated.

Capacitor problem (Fig. 5).- The capacitor in this problem could be viewed as a series of capacitors whose plates were separated by a small distance. The equivalent capacitance could be found by adding the capacitance of each individual capacitor reciprocally, i.e., $\frac{1}{C_{e q}}=\frac{1}{C_{1}}+\frac{1}{C_{2}}+\cdots$, which became $\frac{1}{C_{e q}}=\int \frac{1}{d C}$ when the separation between the plates became infinitesimally small. This problem demanded more than just integrating the infinitesimal quantities to obtain the total quantity. It also required an understanding of integration in association with the physical situation of the problem.

Out of 12 students who attempted the capacitor problem, only two students spontaneously recognized that they had to integrate $\frac{1}{d C}$. The other 10 students integrated $d C$ and got the integral $C=\int d C=\int_{0}^{L} \varepsilon \frac{A(x)}{d x}$. These students immediately recognized that this integral had $d x$ in the denominator, so they attempted to bring $d x$ to the numerator although they could not give a reason why they could do that. The interviewer had to give hints to cue students' attention to the arrangement of the capacitors. The following excerpt is typical in this situation.

Aaron: ... since $L$ is going to turn into $d x$ I think ... but to make that $\ldots$ it should be $d x$ in the denominator ... [writes $\int_{0}^{L} \varepsilon \frac{\frac{\pi d^{2}}{4}}{d x}$ and then flips the integrand]

Interviewer: Why did you flip it?

Aaron: Well, so that $d x$ is in the numerator.

Interviewer: You must have a reason for flipping the integrand.

Aaron: Oh, okay ...

Interviewer: What does your integrand mean?

Aaron: Like if you slice it up it's just one of the slices.

Interviewer: Okay, but when you add up capacitance, you must know how the capacitors are connected, that is, in parallel or in series.

Aaron: Um ... it doesn't say.

Interviewer: Look at how the plates are arranged.

Aaron: Um ...

Interviewer: You should draw some of the fictitious plates to see how they are arranged.

Aaron: [draws the plates] Okay ... so ... they are in series, aren't they?
Interviewer: Yes, and what is the equation for capacitors in series?

Aaron: It's the one over thing.

Interviewer: So how should you integrate in this problem?

Aaron: Well ... because integral means sum ... and I have ... so the integral is ... [writes $\int \frac{1}{d C}$ ]

In this excerpt, Aaron indicated an understanding of the meaning of the integrand, the structure of the integrand (i.e., $d x$ must be in the numerator), and the formula for capacitors in series. However, he was unable to recognize that the capacitors were in series until he drew the fictitious plates between the two plates of the capacitor. Similar situations also occurred with other students who integrated $d C$. This evidence suggested that students' lack of visualization of the physical scenario might account for their disregard of how the quantity must be accumulated.

\section{Computing the integral}

The last step in applying integration to physics problems is to compute the integral set up in the previous three steps. This was expected to be an easy task for students because they had practiced computing integrals in their calculus courses. However, students still had some difficulties with computing the integrals in our interview problems.

Charged arch problem (Fig. 1).-Upon having the integral for the electric field due to the arch $E=\int \frac{1}{4 \pi \varepsilon_{0}} \frac{d q}{r^{2}} \times$ $\cos \theta, 13$ out of 15 students were unable to recall the relation $d q=\lambda d s$ between the charge element $d q$ and the length $d s$ of that element along the arch. Eleven out of 15 students could not relate infinitesimal length of the arc to the infinitesimal angle it subtended at the center: $d s=r d \theta$. After the variable conversion, the resulting simplified integral was $\int_{-\pi / 2}^{\pi / 2} \cos ^{2} \theta d \theta$. All 15 students needed to be given the equation $\cos ^{2} \theta=\frac{1}{2}(1+\cos 2 \theta)$ and two of them needed assistance in computing the integral explicitly.

Charged rod problem (Fig. 2).-We found that students' difficulties with computing the integral in this problem were due to students' inability to interpret the physical meaning of symbols. Twelve out of 15 students interpreted $r$ in Coulomb's law as "radius," so they were unable to decide whether $r$ was a constant or a variable in the integral. The charged rod problem came right after the charged arch problem, so all students were then able to write $d q=\lambda d s$, but 11 of them were unable to recognize that $d s=d x$ in this problem.

Cylindrical conductor problem (Fig. 3).-The integral in this problem was very simple so all students were able to compute it without assistance from the interviewer.

Truncated-cone conductor (Fig. 4) and capacitor problems (Fig. 5).-The most difficult part of computing this integral was to figure out the expression for the crosssectional area as a function of position. However, because 
it was not the purpose of the interview to test students' geometric skills, the expression for $A(x)$ was provided to the students if they failed to get it after a few attempts. The resulting simplified integral was $\int_{0}^{L} \frac{d x}{\{D+[(d-D) / L] x\}^{2}}$, where $D, d, L$ were constants. Only two students succeeded in computing this integral using substitution. Others needed to be given the result of the integral. In the truncated-cone conductor problem, one student set the limits of the integral as $d$ and $D$ (i.e., the diameters of the conductor at two ends) based on the fact that the diameter was changing. The same error was made by five students when solving the capacitor problem, including those who had the correct limits for the integral in the truncated-cone conductor problem.

Current problem (Fig. 6).- - The most difficult part of computing the integral in this problem was to write the differential cross-sectional area $d A$ in terms of the distance $r$ from the center of the wire. Asking students to take the derivative of the cross-sectional area $A=\pi r^{2}$ helped students derive the expression $d A=2 \pi r d r$. The resulting integral was very simple, so all students were able to compute it.

In summary, we found that students encountered a number of difficulties in computing the integrals in physics problems. Some of these difficulties could be attributed primarily to students' misunderstanding of the physical meaning of symbols in the integrals. Other difficulties arose when students could not recall basic mathematical equations. A few students still had difficulties determining the limits of the integrals. Many students were unable to compute mathematical integrals.

\section{CONCLUSION}

In this study, we took a close look at students solving problems involving integration in the context of electricity. We found that students' failure in applying integration to our interview problems occurred when students set up the expressions for the infinitesimal quantities and accumulated those quantities using integral. These difficulties might be attributed primarily to students' inability to interpret the meaning of the infinitesimal term $d x$ in the integral and to students' disregard of how the quantities must be added up. A few students still had difficulties recognizing when an integral was needed in a problem. Students also had difficulties in computing the integrals they had set up, mostly because they were unable to interpret the physical meaning of the symbols and invoke basic mathematical equations.

We answer our research question: What are the common difficulties that students encounter when solving problems in electricity involving integration? Students generally did not have significant difficulty recognizing the need for integration in a problem. However, students did have significant difficulties setting up and computing the desired integral. These difficulties included setting up an incorrect expression for the infinitesimal quantity and/or accumulating the infinitesimal quantities in an inappropriate manner. Determining the limits of the integrals, relating variables in an integral, and computing the integrals algebraically were also difficulties faced by some of the students.

These findings align with those from other research on students' difficulties with integration. We found that the nonconstant quantity given, either mathematically (e.g., resistivity as a function of position, charge distribution as a function of angle) or pictorially (e.g., figure of a conductor with changing diameter), in the problem statement was the cue for most students to think of integration in a problem. This finding supports the conclusion of Meredith and Marrongelle [8] that the most common resource that students use to cue integration is the dependence cue. However, the dependence cue, as pointed out by Meredith and Marrongelle, is only helpful when the nonconstant quantity is a density or a rate of change. This finding also aligns with the fact that many students in our study failed to set up the correct integral in problems involving nonconstant quantities which were not rates of change (e.g., resistivity, diameter).

Although most of the students indicated an understanding of integration as an accumulating process, they were not confident in carrying out the process and needed detailed guidance from the interviewer. Some of the students had difficulties determining the limits of integral. These observations are similar to those described by Cui et al. [2].

Our study extends the literature on students' use of integration in physics problem solving. We found that the major difficulties students encountered when attempting to set up an integral in a physics problem were due to students' inability to understand the infinitesimal term in the integral and failure to understand the notion of accumulation of an infinitesimal quantity.

Meredith and Marrongelle [8] suggested that the partsof-a-whole symbolic form was a powerful and flexible resource to cue integration and proposed instructional strategies to promote students' use of this recourse as a cue for integration in physics problems. Our study points out that setting up a correct integral in a physics problem requires more than recognizing the need for an integral. It also requires setting up the correct expression for the infinitesimal quantity that each "part" represents and accumulating that quantity in a correct manner. There were several students in our interviews who mentioned the sum of infinitesimally small elements (although they did not use that terminology) at some point while solving the problems, indicating that they had a rough idea of the parts-ofa-whole resource, but then set up the incorrect expression for the "part" or did not pay attention to how the "parts" should be added up. So we expand upon the conclusion of Meredith and Marrongelle that although the parts-of-awhole symbolic form is the most powerful and flexible 
way to think of integration, it does not guarantee the correctness of the integral that is set up.

\section{LIMITATIONS AND FUTURE WORK}

The research methodology used in this study was individual interview. This method allowed us to gain detailed insight into students' performance on the problems and also enabled us to interview the same students several times on different topics during the semester. On the other hand, the individual interview method limited the number of student participants in the study. There were only 15 students in our study compared to more than 200 students enrolled in the course. Because of this fact, the major limitation of this study is the generalizability of its findings.

Based on our interview findings, we plan to develop tutorial materials to address students' difficulties with integration and implement them with all of the students in the course (usually around 200+ students) in future semesters when the course is offered to test the effects of those materials in helping students learn to solve physics problems involving integration. As discussed in this paper, the major challenges students faced when solving problems involving integration were in setting up the expression for the infinitesimal quantity and accumulating those quantities in an appropriate manner. Therefore, our tutorials will focus on helping students learn the meaning of the infinitesimal quantity (e.g., $d x, d r, d \theta, \ldots$ ) in the integral and the accumulation process underlying the integral. Our tentative strategy is to use a sample related problem segmented into a sequence of several smaller exercises. The first exercise asks students to calculate the total value of a physical quantity of some individual objects (e.g., the equivalent resistance of a few separate resistors, the equivalent capacitance of a few separate capacitors). The follow-up exercises are variations of the first exercise. In these exercises, the individual objects evolve to become infinitesimal parts of a larger object. We hypothesize that by solving these exercises students might learn how the total quantity of an object becomes an infinitesimal quantity of a larger object and how a sum becomes an integral.

\section{ACKNOWLEDGMENTS}

We gratefully acknowledge the contributions of Dr. Elizabeth Gire, currently at University of Memphis, for her participation in the discussions on the design of the interview protocols used in this study. This work is supported in part by U.S. National Science Foundation Grant No. 0816207.
[1] J. Tuminaro and E. Redish, Understanding students' poor performance on mathematical problem solving in physics, in Proceedings of the Physics Education Research Conference, 2004 (unpublished).

[2] L. Cui, A. Bennett, P. Fletcher, and N. S. Rebello, Transfer of learning from college calculus to physics courses, in Proceedings of the Annual Meeting of the National Association for Research in Science Teaching, 2006 (unpublished).

[3] L. C. McDermott, Oersted Medal Lecture 2001: Physics education research: The key to student learning, Am. J. Phys. 69, 1127 (2001).

[4] A. Orton, Students' understanding of integration, Educ. Stud. Math. 14, 1 (1983).

[5] F. R. Yeatts and J.R. Hundhausen, Calculus and physics: Challenges at the interface, Am. J. Phys. 60, 716 (1992).

[6] T. Grundmeier, J. Hansen, and E. Sousa, An exploration of definition and procedural fluency in integral calculus, Primus 16, 178 (2006).

[7] P.W. Thompson and J. Silverman, The concept of accumulation in calculus, in Making the Connection: Research and Teaching in Undergraduate Mathematics, edited by M. Carlson and C. Rasmussen (Mathematical Association of America, Washington, DC, 2007).
[8] D. C. Meredith and K. A. Marrongelle, How students use mathematical resources in an electrostatics context, Am. J. Phys. 76, 570 (2008).

[9] B. L. Sherin, How students understand physics equations, Cogn. Instr. 19, 479 (2001).

[10] C. Wallace and S. Chasteen, Upper-division students' difficulties with Ampère's law, Phys. Rev. ST Phys. Educ. Res. 6, 020115 (2010).

[11] C. Manogue, K. Browne, T. Dray, and B. Edwards, Why is Ampère's law so hard? A look at middle-division physics , Am. J. Phys. 74, 344 (2006).

[12] L.P. Steffe, The Teaching Experiment Methodology in a constructivist research program, in Proceedings of the Fourth International Congress on Mathematical Education, Boston, MA, 1983 (unpublished).

[13] L.P. Steffe and P.W. Thompson, Teaching Experiment Methodology: Underlying principles and essential elements, in Research Design in Mathematics and Science Education, edited by R. Lesh and A. E. Kelly (Erlbaum, Hillsdale, NJ, 2000), pp. 267-307.

[14] P. V. Engelhardt, E. G. Corpuz, D. J. Ozimek, and N. S. Rebello, The Teaching Experiment-What it is and what it isn't, in Proceedings of the Physics Education Research Conference, Madison, WI, 2003 (unpublished). 